\title{
Avaliação do conhecimento sobre higiene bucal dos responsáveis por crianças de 0-6 anos de idade
}

\section{Evaluation of Knowledge About Oral Hygiene From Primary Caretakers of Children Aged 0-6 Years Old}

\author{
SABRINA Demar \\ Odontolanda pela Faculdade da Serra Gaúcha (FSG), Caxias do Sul/RS - Brasil \\ CAmila Marques \\ Odontolanda pela Faculdade da Serra Gaúcha (FSG), Caxias do Sul/RS - Brasil.
}

JoAnna TAtith Pereira

Mestre em Odontopediatria pela Universidade Federal do Rio Grande do Sul (UFRGS), Porto Alegre/RS - Brasil. Docente do curso de Odontologia da Faculdade da Serra Gaúcha (FSG), Caxias do Sul/RS - Brasil

Renata Shlesner Oliveira Mestre em Odontopediatria pela Universidade Federal do Rio Grande do Sul (UFRGS), Porto Alegre/RS - Brasil. Docente do curso de Odontologia da Faculdade da Serra Gaúcha (FSG), Caxias do Sul/RS - Brasil

Stefanie Bressan Werle Mestre em Odontopediatria pela Universidade Federal de Santa Maria (UFSM), Santa Maria/RS - Brasil. Docente do curso de Odontologia da Faculdade da Serra Gaúcha (FSG), Caxias do Sul/RS - Brasil.

\section{RESUMO}

Objetivo: Avaliar o conhecimento acerca de cuidados bucais dos cuidadores primários das crianças atendidas na clínica de Odontopediatria de uma faculdade no sul do Brasil e sua relação com a prevalência e severidade de cárie e com a condição periodontal (gengivite) dessas crianças. Métodos: Um questionário semiestruturado foi aplicado aos cuidadores primários das crianças com idade entre 0 e 6 anos que procuraram atendimento na Faculdade da Serra Gaúcha (FSG) no período do estudo. As crianças foram examinadas em relação à cárie conforme os escores do ICDAS (International Caries Detection and Assesment System), além de IPV (Índice de Placa Visível) e ISG (Índice de Sangramento Gengival). O questionário incluiu perguntas referentes ao cuidado bucal da criança, como idade e motivo da primeira consulta odontológica, frequência de escovação, quem realiza a escovação, uso de dentifrício fluoretado e sobre o uso regular de fio dental. As variáveis sociodemográficas (renda e escolaridade do responsável) também foram coletadas. Para a análise estatística, os testes Qui-quadrado e Correlação de Sperman foram utilizados com um nível de significância de $5 \%$. Resultados: Um total de 55 crianças com idade média de 4,8 anos $( \pm 1,4)$ participaram do estudo. A prevalência de cárie foi de $92 \%$, enquanto que $76,1 \%$ das crianças apresentaram gengivite. O IPV foi positivo em mais de $20 \%$ dos sítios dentais em $65 \%$ das crianças. Metade dos pais já havia recebido informações sobre a higiene bucal da criança. Em torno de $80 \%$ dos pais relataram realizar a higiene bucal diariamente, e quase $60 \%$ disseram fazer a higiene mais de duas vezes ao dia. O dentifrício fluoretado foi introduzido antes dos 3 anos de idade em aproximadamente $75 \%$ dos pacientes. Menos de $50 \%$ das consultas foram motivadas pela prevenção. O nível de escolaridade dos responsáveis não apresentou associação estatisticamente significativa com as variáveis clínicas. A renda familiar apresentou correlação negativa com o IPV $(c s-0,324 p=0,028)$, perfil do paciente em relação à cárie $\left({ }_{c s}-0,289 p=0,042\right)$ e severidade da cárie $\left({ }_{c s} 0,401 p=0,004\right)$. A severidade da cárie mostrou correlação com o IPV $\left({ }_{c s} 0,355 p=0,017\right)$ e com o perfil do paciente em relação à cárie $\left({ }_{c s} 0,650 p<0,001\right)$. A idade da primeira consulta odontológica apresentou correlação com o ISG $(\operatorname{cs} 0,299 p=0,043)$ e IPV $(\operatorname{cs} 0,341 p=0,020)$ da criança. Conclusão: $O$ presente estudo mostrou uma alta prevalência de cárie e gengivite em crianças de 0-6 anos. O conhecimento sobre cuidados bucais dos responsáveis parece limitado, especialmente quanto à forma de realização da escovação e importância de consulta de prevenção.

Palavras-chave: Odontopediatria; cárie dentária; higiene bucal; conhecimento; Gengivite; Fio dental. 


\begin{abstract}
Objective: To evaluate the knowledge about oral hygiene from primary caretakers of children attending in the clinic of Faculty of Serra Gaucha and its relationship with the prevalence and severity of dental caries and gingivitis. Method: A questionnaire with questions about the oral hygiene was applied to caretakers of children between 0-6 years of age attended at Faculty of Serra Gaucha. A clinical examination was performed in children to detect caries lesions (ICDAS - International Caries Detection and Assessment System), and VPI (Visible Plaque Index) and GBI (Gingival Bleeding Index). The questionnaire included questions related to the oral care of the child, such as age and reason for first dental visit, brushing frequency, who performs brushing, use of fluoride dentifrice and regular flossing. Sociodemographic variables (income and education level) were also collected. For statistical analysis, the chi square test and Sperman's correlation ( $\left.{ }_{\mathrm{sC}}\right)$ were used. Results: A total of 55 children with an average age of 4.8 years $( \pm 1.4)$ participated in the study. The prevalence of caries was $92 \%$, while $76.1 \%$ of children had gingivitis. The VPI was positive in more than $20 \%$ of dental sites in $65 \%$ of children. Half of caretakers had received information about the oral hygiene of the child. Approximately $80 \%$ of them reported doing daily oral hygiene, and nearly $60 \%$ performed oral hygiene more than 2 times per day. Fluoride toothpaste was introduced before 3 years of age in approximately $75 \%$ of patients. Less than $50 \%$ of dental visits were motivated for prevention. The education level of caretakers showed no statistically significant association with clinical variables. Family income was negatively correlated with the VPI $\left({ }_{s c}-0.324 p=0.028\right)$, patient's profile in relation to caries $\left({ }_{s c}-0.289 p=0.042\right)$ and severity of dental caries $\left({ }_{\mathrm{Sc}}-0.401 \mathrm{p}=0.004\right)$. The severity of caries correlated with VPI $\left({ }_{\mathrm{Sc}} 0,355 \mathrm{p}=0.017\right)$ and patient profile in relation to caries $\left({ }_{S C} 0,650 p<0.001\right)$. The age at first dental visit was correlated with $\mathrm{GBI}$ $\left({ }_{S C} 0,299 p=0.043\right)$ and VPI $\left({ }_{S C} 0,341 p=0.020\right)$. Conclusion: This study showed a high prevalence of caries and gingivitis in children aged 0-6 years. Knowledge about oral care of caretakers appears to be limited, especially about brushing performance and importance of prevention visits.
\end{abstract}

Key-words: Pediatric dentistry; dental caries; oral hygiene; knowledge; gingivitis; dental floss.

\section{INTRODUÇÃO}

A cárie dentária é a infecção oral mais comum no mundo. ${ }^{1}$ De acordo com o último levantamento epidemiológico de saúde bucal realizado no Brasil, ${ }^{2}$ crianças em idade préescolar apresentam uma prevalência de cárie próxima a $50 \%$. A lesão de cárie é resultante das flutuações de $\mathrm{pH}$ no biofilme dental, ${ }^{3}$ que levam à desmineralização dos tecidos duros do órgão dental.

Outra doença bucal altamente prevalente em crianças é a gengivite, ${ }^{4}$ sendo o acúmulo de biofilme dental o principal fator de risco para o seu desenvolvimento. ${ }^{5}$ Uma vez que a gengivite é diagnosticada em crianças, precisa ser tratada, visto que além de ser um sítio de inflamação, pode representar um risco para o desenvolvimento da periodontite quando tais indivíduos se tornam adultos. ${ }^{6}$

O principal fator para a prevenção da cárie e gengivite inclui a remoção mecânica do biofilme. $^{7-8-9}$ Aliado ao controle do biofilme, a presença de flúor na cavidade bucal tem um efeito importante na prevenção de cárie. ${ }^{10}$ Isso porque o flúor é capaz de diminuir a desmineralização durante as quedas de $\mathrm{pH}$ e, quando o pH é reestabelecido, acelerar a remineralização com a formação de fluorapatita, mineral menos solúvel que a hidroxiapatita, tornando a superfície mais resistente aos futuros desafios ácidos. ${ }^{11}$ Por isso, é recomendado que a higiene bucal da criança, desde a erupção do primeiro dente, seja realizada com uso de escova multicerdas, dentifrício fluoretado ${ }^{11-12}$ e uso de fio dental, quando necessário. ${ }^{13}$

A higiene bucal em crianças até 10 anos de idade é de responsabilidade dos cuidadores primários, por isso é essencial que os pais compreendam a sua importância e sejam capazes de mantê-la, como parte da saúde geral de si e de seus filhos. ${ }^{14}$ Além disso, a infância é um período crítico de aquisição de novos hábitos, que poderão refletir no futuro padrão e comportamentos em saúde. ${ }^{15}$ 
Alguns fatores socioeconômicos, como renda familiar, ${ }^{16}$ ingresso precoce da criança na escola, nível de escolaridade dos pais, acesso à informação em relação à saúde, bem como o acesso à saúde publica ${ }^{17}$ estão associados à prevalência e severidade das doenças bucais.

Este trabalho teve como objeto avaliar a) o nível de informação sobre cuidados bucais dos cuidadores primários das crianças atendidas na clínica de odontopediatria de uma faculdade no sul do Brasil e b) a relação dos dados referentes ao nível de conhecimento com a prevalência de cárie e gengivite, controle do biofilme e as variáveis sociodemográficas.

\section{Metodologia}

Este estudo transversal foi constituído por uma amostra de conveniência. Os cuidadores primários de crianças entre 0 a 6 anos de idade que procuraram atendimento na Clínica de Odontopediatria da Faculdade da Serra Gaúcha (FSG), Caxias do Sul-RS, Brasil, entre os meses de outubro de 2014 e maio de 2015 foram convidados a participar.

O presente estudo foi aprovado pelo Comitê de Ética e Pesquisa (CEP) da Faculdade da Serra Gaúcha (n. 834.541) e os dados foram coletados após consentimento formal dos responsáveis participantes por meio da assinatura do Termo de Consentimento Livre e Esclarecido.

As informações foram coletadas a partir de um questionário semiestruturado aplicado ao cuidador primário por dois pesquisadores treinados. O preenchimento do questionário ocorreu no primeiro dia de atendimento da criança, exceto quando o acompanhante não era o cuidador direto da criança (nessa condição, o questionário foi aplicado em outro momento do tratamento). Esse questionário incluiu duas partes, a primeira com questões abordando as características sociodemográficas da criança como renda e escolaridade do responsável. A segunda parte incluiu questões sobre os cuidados com a higiene bucal da criança, como a frequência de escovação, quem realiza a higiene bucal, qual o tipo e a quantidade de dentifrício utilizado, idade na primeira consulta odontológica, o motivo da mesma e o uso regular de fio dental.

Para adequação da linguagem e validação do questionário, o mesmo foi aplicado a 10 responsáveis por pacientes atendidos na clínica de odontopediatria da FSG. Após esse processo, algumas questões foram adaptadas para serem compreendidas facilmente pelos participantes.

O método para a detecção de lesão de cárie utilizado foi o visual-tátil, conforme os escores do ICDAS (International Caries Detection and Assesment System). ${ }^{18} \mathrm{O}$ exame foi realizado após deplacagem profissional e efetuado com uso da luz do refletor e de isolamento relativo. O exame clínico foi feito por três examinadores experientes. O perfil do paciente em relação à carie foi categorizado em livre de cárie/cárie inativo e cárie ativo. A severidade da doença foi definida pela ausência ou presença de lesão cavitada.

Para a aferição do controle do biofilme no paciente, foi realizado o Índice de Placa Visível (IPV). O método utilizado para a detecção de gengivite foi o Índice de Sangramento Gengival (ISG). ${ }^{19}$ Ambos os exames foram feitos com auxílio do jato de ar e sob iluminação do refletor. Os alunos do quinto, sexto e oitavo semestres do curso do Curso de Odontologia da FSG realizaram os exames sob supervisão de professores experientes em odontopediatria.

A análise estatística foi realizada usando o programa SPSS v.18, com um nível de significância de 5\%. Para avaliar as variáveis quantitativas ou qualitativas ordinais, o teste de correlação de Spearman $\left({ }_{c S}\right)$ foi utilizado. O teste qui-quadrado foi aplicado para avaliar as variáveis qualitativas. Os exames, quando 
não realizados (consulta de urgência sem retorno do paciente/bebês edêntulos), foram tratados como dados não incluídos. Para a análise descritiva dos dados, algumas variáveis foram categorizadas.

\section{Resultados}

Um total de 55 crianças com idade média de 4,8 anos $( \pm 1,4)$ participou do estudo. As variáveis sociodemográficas e o motivo da procura à Clínica de Odontopediatria da FSG estão descritos na Tabela 1.

Tabela 1 - Descrição das variáveis sociodemográficas referentes à família dos pré-escolares e o motivo da consulta odontológica.

\begin{tabular}{lcc}
\hline Variáveis sociodemográficas & $\mathbf{n}$ & $\%$ \\
\hline Renda (em R\$) & 11 & 20 \\
$<1000,00$ & 27 & 49,1 \\
$>1000,00<2000$ & 17 & 30,9 \\
$>2000,00$ & & \\
\hline Escolaridade materna & 4 & 7,3 \\
Fundamental incompleto & 12 & 21,8 \\
Fundamental completo & 33 & 60 \\
Médio completo & 6 & 10,9 \\
Superior completo & & \\
\hline Motivo da consulta & 23 & 41,8 \\
Revisão & 24 & 43,6 \\
Dor/cárie & 8 & 14,6 \\
Estética & & \\
\hline
\end{tabular}

A Tabela 2 apresenta os dados referentes ao conhecimento acerca da higiene bucal da criança relatado pelos responsáveis. Um total de $92 \%$ dos pacientes apresentou pelo menos uma lesão de cárie (ativa/inativa), enquanto $76,1 \%$ apresentaram gengivite. Os dados clínicos coletados dos pacientes estão descritos na Tabela 3.

O nível de escolaridade dos responsáveis não apresentou associação estatisticamente significativa com as variáveis clínicas. A renda familiar mostrou correlação negativa com IPV $\left({ }_{c s}-0,324 p=0,028\right)$, perfil do paciente em relação à cárie $\left({ }_{c s}-0,289 p=0,042\right)$ e severidade da cárie ( $0,401 \mathrm{p}=0,004)$.
Tabela 2 - Conhecimentos sobre higiene bucal em préescolares relatados pelos responsáveis.

\begin{tabular}{|c|c|c|}
\hline Conhecimento dos responsáveis & $\mathbf{n}$ & $\%$ \\
\hline \multicolumn{3}{|l|}{$\begin{array}{l}\text { Recebeu informação sobre a higiene } \\
\text { bucal da criança }\end{array}$} \\
\hline Não & 28 & 50,9 \\
\hline $\operatorname{Sim}$ & 27 & 49,1 \\
\hline \multicolumn{3}{|l|}{$\begin{array}{l}\text { A limpeza bucal da criança é realizada } \\
\text { diariamente }\end{array}$} \\
\hline $\operatorname{Sim}$ & 44 & 80,0 \\
\hline Não & 11 & 20.0 \\
\hline \multicolumn{3}{|l|}{$\begin{array}{l}\text { Quem realiza a limpeza na maioria das } \\
\text { vezes }\end{array}$} \\
\hline Criança & 18 & 32,7 \\
\hline Responsável & 23 & 41,8 \\
\hline Ambos & 14 & 25,5 \\
\hline \multicolumn{3}{|l|}{ Frequência de escovação diária } \\
\hline$<2$ vezes & 23 & 41,8 \\
\hline$>2$ vezes & 32 & 58.2 \\
\hline \multicolumn{3}{|l|}{$\begin{array}{l}\text { Qual a quantidade de dentifrício } \\
\text { utilizada }\end{array}$} \\
\hline Figura 1 TIIII & 13 & 23,6 \\
\hline Figura 2 & 22 & 40,0 \\
\hline Figura $3 \quad$ MII $\sim$ & 13 & 23,6 \\
\hline Fiqura 4 & 7 & 12,8 \\
\hline \multicolumn{3}{|l|}{$\begin{array}{l}\text { Recebeu Informação sobre a } \\
\text { quantidade de dentifrício }\end{array}$} \\
\hline Não & 38 & 69,1 \\
\hline Sim & 17 & 30.9 \\
\hline \multicolumn{3}{|l|}{$\begin{array}{l}\text { Idade que começou a utilizar dentifrício } \\
\text { fluoretado }\end{array}$} \\
\hline$<3$ anos & 41 & 74,6 \\
\hline$>3$ anos & 8 & 14,5 \\
\hline Não usa & 6 & 10.9 \\
\hline \multicolumn{3}{|l|}{ Usa fio dental regularmente } \\
\hline Não & 34 & 61,8 \\
\hline Sim & 21 & 38,2 \\
\hline \multicolumn{3}{|l|}{$\begin{array}{l}\text { Idade da primeira consulta } \\
\text { odontológica da criança }\end{array}$} \\
\hline Até completar o $1^{\circ}$ ano de vida & 11 & 20,0 \\
\hline Entre o $1^{\circ}$ e o $3^{\circ}$ ano de vida & 24 & 43.6 \\
\hline Após o $3^{\circ}$ ano de vida & 20 & 36.4 \\
\hline
\end{tabular}

Entre as variáveis referentes ao nível de conhecimento relatado pelos responsáveis, a idade da primeira consulta odontológica apresentou correlação moderada com o ISG $\left({ }_{c s} 0,299 p=0,043\right)$, IPV $\left({ }_{c s} 0,341 p=0,020\right)$ e severidade da cárie $\left({ }_{c s} 0,328 p=0,028\right)$.

Aseveridade da cárie apresentou correlação moderada com o IPV $\left({ }_{c s} 0,355 p=0,017\right)$ e uma forte correlação com o perfil do paciente em relação à cárie $\left({ }_{c s} 0,650 p<0,001\right)$. 
Tabela 3 - Distribuição dos desfechos clínicos coletados.

\begin{tabular}{|c|c|c|}
\hline Variáveis clínicas & $\mathbf{n}$ & $\%$ \\
\hline \multicolumn{3}{|c|}{ Perfil do paciente em relação à doença cárie } \\
\hline Livre de cárie/Cárie inativa & 7 & 13,7 \\
\hline Cárie ativo & 44 & 86,3 \\
\hline \multicolumn{3}{|l|}{ Severidade da lesão cárie } \\
\hline não cavitada & 11 & 23,9 \\
\hline cavitada & 35 & 76,1 \\
\hline \multicolumn{3}{|l|}{ İndice de sangramento gengival } \\
\hline$<20 \%$ & 32 & 69,6 \\
\hline$>20 \%$ & 14 & 30,4 \\
\hline \multicolumn{3}{|l|}{ Índice de placa visível } \\
\hline$<20 \%$ & 16 & 34,7 \\
\hline$>20 \%$ & 30 & 65,2 \\
\hline
\end{tabular}

\section{Dıscussão}

Um pouco mais da metade $(50,9 \%)$ dos entrevistados neste estudo não teve acesso à informação de como deve ser feita a higiene bucal do seu filho, o que demonstra uma falha na atuação dos profissionais de saúde, especialmente dentistas e pediatras que assistem a essa população e até mesmo a falta de acesso a eles. Uma porção significativa $(32,7 \%)$ dos entrevistados respondeu que a criança realiza a escovação sozinha. Esse relato demonstra o equívoco na informação, uma vez que a escovação feita por crianças menores de 10 anos de idade pode ser ineficiente, em virtude da baixa habilidade manual, além da falta de motivação proporcionada pelos responsáveis e pelo grau de responsabilidade que apresentam sobre a sua higiene bucal e corporal. ${ }^{14,20}$

A Academia Americana de Pediatria recomenda como medida preventiva que a primeira consulta ao dentista deve ocorrer até o primeiro ano de vida. ${ }^{21}$ Entretanto, uma mínima porcentagem (20\%) da amostra segue essa recomendação. Ao contrário, foi visto que o principal motivo de procura de atendimento odontológico ocorreu em casos de dor/cárie $(43,6 \%)$. Esse índice retrata uma situação alarmante, pois a cárie tem um impacto negativo na vida das crianças, tanto socialmente quanto nas funções diárias, como a mastigação. ${ }^{22}$ Outros estudos também demonstraram uma prevalência preocupante (11-16\%) de pais que procuram $\mathrm{o}$ atendimento odontológico apenas quando a criança se queixa de dor. ${ }^{23-}$ 24 Esse cenário de procura odontológica somente para a intervenção, quando a doença já está instalada, leva ao ciclo restaurador repetitivo. ${ }^{25}$ A detecção precoce dos fatores etiológicos, bem como o diagnóstico precoce da cárie, proporciona ao paciente tratamentos menos invasivos e menor perda de estrutura dentária, o que deve ser sempre buscado pelos pacientes e profissionais nas consultas periódicas de prevenção. ${ }^{23}$ Neste estudo, uma correlação foi encontrada entre a idade na primeira consulta e os índices de IPV e ISG, ou seja, quanto antes a consulta é realizada, tanto menores esses índices, demonstrando o efeito preventivo dessa consulta.

Embora a maioria dos cuidadores primários tenha relatado que a limpeza dos dentes da criança é realizada diariamente $(80 \%)$ e grande parte $(58,2 \%)$ tenha respondido que a fazem mais de duas vezes ao dia, corroborando com estudos prévios realizados com amostras maiores, ${ }^{26,27}$ esses resultados positivos não foram estendidos aos exames que avaliaram o controle do biofilme (IPV) e gengivite (ISG). A influência da mídia, que menciona que a escovação deve ser feita três vezes ao dia, pode ter acarretado em uma resposta viciada por parte dos entrevistados. Além disso, já foi relatado a baixa concordância entre a escovação observada e o relato do cuidador primário. ${ }^{28}$

Além disso, foi observado que $61,8 \%$ das crianças participantes não fazem o uso regular de fio dental. Embora alguns pacientes tenham espaços interdentais, o fio dental deve ser utilizado regularmente quando existe ponto de contato interdental sem alcance das cerdas da escova. Esse hábito não praticado também por parte dos adultos deve-se também por 
esses não estarem cientes da importância do uso correto do fio dental que, de fato, é indispensável para a manutenção de saúde periodontal. ${ }^{29}$

Os resultados dos exames de IPV relativamente altos $(65,2 \%$ apresentaram placa visível em mais de $20 \%$ das superfícies dentais) demonstram uma falha no controle do fator etiológico da cárie e gengivite. Esse descontrole do biofilme dental possivelmente justifica os altos índices de gengivite encontrados nas crianças $(76,1 \%)$, em que quase um terço da amostra $(30,4 \%)$ apresentou sangramento em mais do que $20 \%$ dos sítios examinados. A categorização do IPV e ISG em mais ou menos de $20 \%$ refere-se à não relevância clínica dessas variáveis quando abaixo de $20 \%{ }^{30}$

Assim como a gengivite, a cárie também é resultado do controle deficiente do biofilme dental, e ambas as doenças têm se mostrado altamente prevalentes na população infantil brasileira. ${ }^{2-31} \mathrm{O}$ presente estudo, realizado com crianças de 0 a 6 anos de idade, encontrou uma prevalência de $92 \%$ da doença, enquanto outros autores apontam uma prevalência próxima aos 50\%. ${ }^{12-16-17-32}$ Essa maior expressão na prevalência de cárie pode estar relacionada à amostra, que é constituída por pacientes que procuraram $\mathrm{o}$ atendimento odontológico, diferentemente de estudos com base populacional. Além disso, o método de detecção de cárie foi o ICDAS, diferentemente da maioria dos outros trabalhos, que usam como método o CPO-D, que subestima a prevalência de cárie quando comparado ao método usado neste trabalho. ${ }^{33}$ Quando consideramos apenas as lesões cavitadas em dentina, $76,1 \%$ das crianças apresentavam pelo menos um dente acometido com essa condição.

Para a prevenção da cárie, a remoção mecânica do biofilme com o uso de escova multicerdas e dentifrício fluoretado é um fator determinante..$^{7-8-9} \mathrm{Em}$ relação ao uso de dentifrício fluoretado, $10,9 \%$ relataram nunca ter usado e $14,5 \%$ só após os 3 anos de idade. No entanto, ainda que quase $75 \%$ tenham respondido que usaram o dentifrício fluoretado antes dos 3 anos de idade, já foi relatado que os responsáveis parecem não conhecer a importância do flúor, e acabam utilizando o dentifrício de 1000ppm (parte por milhão) nas crianças devido à comodidade de já possuir em casa. Embora o uso de dentifrício fluoretado não tenha sido um fator associado à prevalência de cárie neste estudo, o que corrobora com Ota e colaboradores, ${ }^{34}$ revisões sistemáticas apontam que o uso regular de dentifrício de alta concentração (acima de 1000 ppm F) apresenta efeito preventivo de cárie. ${ }^{9}$ A não verificação da associação entre essas variáveis neste estudo pode estar relacionada às discrepâncias entre o que é relatado no questionário e a correspondência cotidiana real para essa família, ${ }^{15-28}$ bem como ao tamanho da amostra.

No presente estudo, não foi observada uma correlação entre o nível de escolaridade dos pais e a incidência de cárie, diferentemente de outros estudos que apontaram a escolaridade materna como um fator de risco para desenvolver a doença. ${ }^{17-32}$ Esse dado pode estar relacionado ao pequeno tamanho da amostra $(n=55)$, bem como à distribuição do nível de escolaridade, em que $70 \%$ dos responsáveis apresentam ensino médio/ superior.

Entre as variáveis sociodemográficas, observou-se que a renda familiar apresentou uma correlação negativa relacionada com o IPV. Dessa forma, quanto menor a renda, tanto maior o índice de IPV encontrado, o que corrobora com os achados de um estudo prévio. ${ }^{31}$

Esse cenário de alta prevalência $\mathrm{e}$ severidade de cárie e gengivite demonstra a necessidade de estabelecer programas que 
visem promover a saúde bucal infantil por meio do controle do biofilme dental pelos cuidadores das crianças.

Algumas prováveis limitações deste estudo foram: a dificuldade de realização do exame de ISG na criança pelos alunos, que pode ter gerado falsos negativos; a possível discrepância entre os relatos ao questionário e à higiene bucal realmente realizada; e a constituição de uma amostra de conveniência.

\section{Conclusão}

O presente estudo mostrou uma alta prevalência de cárie e gengivite em crianças de 0-6 anos de idade que procuraram atendimento na FSG. O nível de conhecimento dos pais foi considerado limitado, e a capacidade de controle de biofilme da criança não foi adequada. Foi observada a necessidade de capacitar os responsáveis para manter adequada higiene bucal e consequente manutenção de saúde bucal da criança. Além disso, é preciso haver uma valorização das consultas de prevenção/manutenção.

\section{REFERÊNCIAS}

1. Jeon JG, Rosalen PL, Falsetta ML, Koo H. Natural products in caries research: current (limited) knowledge, challenges and future perspective. Caries Res. 2011; 45: 243-63.

2. Brasil. Ministério da Saúde. Secretaria de Vigilância em Saúde. Secretaria de Atenção à Saúde. Departamento de Atenção Básica. Coordenação Nacional de Saúde Bucal. SB Brasil 2010. Pesquisa Nacional de Saúde Bucal. Resultados Principais. Brasília: MS, 2011.

3. Kidd E., Fejerskov, O. Changing concepts in cariology: forty years on. Dent Update, 2013, May; 40(4): 277-8, 280-2.

4. Padovani MCRL, Santos MTBR, Sant'anna GR, Guaré RO. Prevalence of oral manifestations in soft tissues during early childhood in Brazilian children. Braz. Oral Res., 2014; 28(1): 1-7.
5. Sarabia MCM, Gutiérrez GR, Olazábal YR. Factores de riesgo em las periodontopatías de escolares. AMC, 2006; 10(5): 4-13.

6. Gomes SC, Romagna R., Rossi V., Corvello PC, Angst PDM. Supragingival treatment as an aid to reduce subgingival needs: a 450-day investigation. Braz. Oral Res., 2014; 28(1): 1-7.

7. Wigen TI, Wang NJ. Tooth brushing frequency and use of fluoride lozenges in children from 1.5 to 5 years of age: a longitudinal study. Community Dent. Oral Epidemiol, 2014; 42(5): 395-403.

8. Aminabadi NA, Ghoreishizadeh A., Ghoreishizadeh M., Oskouei SG, Ghojazadeh M. Can child temperament be related to early childhood caries? Caries Res., 2014; 48: 3-12.

9. Broffitt B., Levy SM, Warren J., Cavanaugh JE. Factors associated with surface-level caries incidence in children aged 9 to 13: the lowa Fluoride Study. J. Public. Health Dent., 2013; 73(4): 304-10.

10. Marinho VC, Worthington HV, Walsh T., Clarkson JE. Fluoride varnishes for preventing dental caries in children and adolescents. Cochrane Database Sys Rev., 2013; 11: CD002279.

11. Buzalaf MA, Pessan JP, Honório HM, Ten cate, JM. Mechanisms of action of fluoride for caries control. Monog. Oral Science, 2011; 22: 97-114.

12. Olatosi OO, Inem V., Sofola OO, Prakash P., Sote EO. The prevalence of early chilldhood caries and its associated risk factors among preschool children referred to a tertiary care institution. Nigerian J. Clin. Pract, 2015; 18(4): 493-501.

13. Teles PR, Teles FRF. Antimicrobial agents used in the control of periodontal biofilms: effective adjuncts to mechanical plaque control? Braz. Oral Res., 2009; 23(1): 39-48.

14. Navarro MLR, Reyes SS, Araujo MEF, Zavala VHGS. Prevalencia y grado de gingivitis asociada a placa dentobacteriana en niños. Revista Electrónica Nova Scientia, 2014; 6 (2) 190-218.

15. Cascaes AM, Peres KG, Peres MA, Demarco FF, Santos I, Matijasevich A., Barros AJD. Validade do padrão de higiene bucal de crianças aos cinco anos de idade relatado pelas mães. Rev. Saúde Pública, 2011; 45(4): 668-75. 
16. Ardenghi MT, Piovesan C., Antunes FJL. Desigualdades na prevalência de cárie dentária não tratada em crianças pré-escolares no Brasil. Saúde Pública, 2013; 47(3): 129-37.

17. Correa-Faria P., Junior MAP, Andrade VGR, Marques SL, Jorge RLM. Factors associated with the development of early childhood caries among Brazilian preschoolers. Braz. Oral Res., 2013; 27(4): 356-62.

18. Ismail AI, Sohn W., Tellez M., Amaya A., Sen A., Hasson $\mathrm{H}$. et al. The International Caries Detection and Assessment System (ICDAS): an integrated system for measuring dental caries. Community Dent. Oral Epidemiol., 2007; 35(3): 170-8.

19. Ainamo J., Bay I. Problems and proposals for recording gingivitis and plaque. Int. Dent. J., 1975; 25(4): 229-35.

20. Sharma S., Yeluri R., Jain AA, Munshi AK. Effect of toothbrush grip on plaque removal during manual toothbrushing in children. J Oral Sci 2012; 54(2): 183-190.

21. Hale KJ. Oral health risk assessment timing and establishment of the dental home. Pediatrics, 2003; 111(5): 1.113-1.116.

22. Feitosa S., Colares V., Pinkham J. The psychosocial effects of severe caries in 4-yearold children in Recife, Pernambuco, Brazil. Cad. Saúde Pública, 2005; 21(5): 1.550-1.556.

23. Moura-Leite FR, Ramos-Jorge ML, Bonanato K., Paiva SM, Vale MP, Pordeus IA. Prevalence, intensity and impact of dental pain in 5-year-old preschool children. Oral Health Prev. Dent., 2008; 6(4): 295-301.

24. Boeira GF, Correa MB, Peres KG, Peres MA, Santos IS, Matijasevich A. et al. Caries is the main cause for dental pain in childhood: findings from a birth cohort. Caries Res., 2012; 46(5): 488-95.

25 . Ericson D. The concept of minimally invasive dentistry. Dent Update, 2007; 34(1): 9-10, 12 4, 17-8.

26. Casanova JF, Vallejo AA, Minaya M., Medina CE, De La Rosa R., Marquez M., Maupomé G. Frequency of tooth brushing and associated factors in mexican schoolchildren six to nine years of age. West Indian Med., 2013; 62(1): 68-72.
27. Wigen TI, Wang NJ. Tooth brushing frequency and use of fluoride lozenges in children from 1.5 to 5 years of age: a longitudinal study. Community Dent. Oral Epidemiol., 2014; 42(5): 395-403.

28. Martins CC, Oliveira MJ, Pordeus IA, Paiva SM. Comparison between observed children's tooth brushing habits and those reported by mothers. BMC Oral Health, 2011; 11(22): 1-7.

29. Silva AMM, Souza GA, Galvão R. Autoeficácia, acúmulo de placa dental e relato de comportamento de higiene oral. Estudos de Psicologia, 2000; 17(2): 62-72.

30. Lang NP, Tonetti MS. Periodontal risk assessment (PRA) for patients in supportive periodontal therapy (SPT). Oral Health Prev Dent., 2003; 1: 7-16.

31. Chiapinotto FA, Ferreira FV, Demarco FF, Corrêa FOB, Masotti AS. Risk factors for gingivitis in a group of Brazilian schoolchildren. J. Public. Health Dent., 2013; 73: 9-17.

32. Kuriakose S., Prasannan M., Remya KC, Kurian J., Sreejith KR. Prevalence of early childhood caries among preschool children in Trivandrum and its associatin with various risck factors. Contem. Clin. Dent., 2015; 6(1): 69-73.

33. Mendes MF, Braga MM, Oliveira BL, Antunes FLJ, Ardenghi MT, Bonecker M. Discriminant validity of the international caries detection and assessment system (ICDAS) and comparability with world health organization criteria in a cross-sectional study. Community Dent. Oral Epidemiol., 2010; 38(5): 398-407.

34. Ota J., Yamamoto T., Ando Y., Aida J., Hirata Y., Arai S. Dental health behavior of parents of children using non-fluoride toothpaste: a cross sectional study. BMC Oral Health, 2013; 14(74): 1-7.

Autor para correspondência:

Stefanie Werle

stewerle@hotmail.com

Submetido em: 13-11-2015

Aceito em: 28-3-2016 